\title{
The DDO short-period binary RV program
}

\author{
Slavek M. Rucinski \\ Department of Astronomy and Astrophysics, University of Toronto
}

September 15, 2005

\begin{abstract}
The short-period binary radial velocity program at the David Dunlap Observatory is described in some detail, with a brief summary on its history. The program is now reaching its 100 -th orbit, with about 40 more orbits remaining within our access to Northern $(\delta>-15)$, bright $(<10 \mathrm{mag})$, short-period $(<1$ day $)$ binary systems. All data are processed uniformly using the Broadening Function (BF) formalism which offers best information extraction and permits analysis of heavily rotationally blended spectra. Many close binaries appear to have visual or spectroscopic companions.
\end{abstract}

Keywords: binary stars, radial velocities, broadening functions

\section{History of the program}

The David Dunlap Observatory (DDO $)^{1}$, located in Richmond Hill, about $25 \mathrm{~km}$ North of the centre of Toronto has been in operation since 1935. The encroachment of the city of Toronto into the neighbouring Richmond Hill, started in the 1960s and becoming very fast in the 1980s forced concentration of the $1.88 \mathrm{~m}$ telescope program on spectral observations only. Influenced by the Great Lakes climate (observing can be done on typically $50 \%$ of partial nights, while the overall efficiency in terms of hours is about 30\%) and the progressively brighter sky, the observatory must seek niches where it can still provide useful research.

Hilmar Duerbeck and the undersigned had been involved in the selection of contact binaries for the Hipparcos program in the early 1980s; all objects fainter than 7.5 had to be explicitly included. When the accurate parallax and proper motion Hipparcos data became available, we realized that the weak point in evaluation of space velocities is a lack of radial velocity data. The late 1990s was the time when the $1.88 \mathrm{~m}$ telescope showed first signs of being under-utilized, so we proposed that the DDO Telescope Operators (TOs) observe several contact binaries which - eventually, after averaging in time of the orbital motion would give some preliminary information on centre-of-mass velocities. Wen Lu was one of the TOs. His interests in contact binaries led to more extensive observations covering full orbital data for a few systems. We published the first results (1999, Paper I; 1999, Paper II; 2000, W Crv)

\footnotetext{
${ }^{1}$ http://www.astro.utoronto.ca/DDO/
}

(C) 2005 Kluwer Academic Publishers. Printed in the Netherlands. 
without much conviction of the undersigned that the program could be done on a systematic basis. Certainly, we did not have any plans to continue it for the next 7 years and to produce more than 100 radial velocity orbits...

\section{Technical details}

The radial velocity spectra are obtained with a spectrograph in the Cassegrain focus of the $1.88 \mathrm{~m}$ telescope. The spectrograph was modern when it started to be used in 1969: It utilizes all-reflection optics and blazed refelction gratings. It was designed for photographic work, but since 1989 it uses a liquid-nitrogen cooled CCD. Our program started when a thick, $19 \mu \mathrm{m} /$ pixel, $1 K \times 1 K$ Thomson chip was the main detector. It continued until 2003 when this chip failed and had to be replaced, first by an identical one and, later, by a $13.5 \mu \mathrm{m} 0.5 \mathrm{~K} \times 2 \mathrm{~K}$ thinned Marconi CCD. For most of our observations, we have used the $1800 \mathrm{l} / \mathrm{mm}$ grating at the MgI "b" triplet $5184 \AA$. The triplet is relatively strong over a wide range of spectral types and can be used for RV determinations from the middle A to middle K spectral types. Very recently, we have replaced the much used (and scratched) $1800 \mathrm{l} / \mathrm{mm}$ grating by a $2160 \mathrm{l} / \mathrm{mm}$ grating, so that - with the smaller pixels and the new grating - our sampling has changed from about $11 \mathrm{~km} / \mathrm{s} / \mathrm{pix}$ to $5.7 \mathrm{~km} / \mathrm{s} /$ pix. The sky at $5184 \AA$ is still quite dark. While the overall brightness at DDO in the V-filter is as high as $17 \mathrm{mag} / \operatorname{arcsec}^{2}$ on dark nights, the $5184 \AA$ region is by a couple of magnitudes darker.

The telescope - spectrograph combination can give for sharp-line stars radial velocity data good to slightly better than $1 \mathrm{~km} / \mathrm{s}$. The accuracy is determined by the internal flexure in the system which limits exposures to below 20-30 minutes. For heavily broadened and blended spectra of short-period binary stars, the accuracy of individual observations depends mostly on processing; it drops to the level of 3 - $10 \mathrm{~km} / \mathrm{s}$ for the primary components and to $5-25 \mathrm{~km} / \mathrm{s}$ for the fainter secondaries. A preliminary description of our program and of its limitations was given in (Rucinski, 2002, Paper VII), where the main method of the data extraction utilizing the Broadening Function formalism is also described.

\section{Broadening Functions}

The Broadening Function (BF) approach is very simple in its principle, but it is rather demanding on the mathematical implementation and 
hence it is not really as easy to use as the Cross Correlation Function (CCF) approach. We define a broadening function, $B$, as a kernel in an integral convolution transformation of a sharp-line spectrum, $S$, into a binary star spectrum, $P$. Given the observed spectra $S$ and $P$, the goal is to determine the broadening kernel $B$ from the integral equation:

$$
P(x)=\int B(u) S(x-u) d u
$$

One of possible operations is to compute the CCF using $S$ and $P$ :

$$
C(z)=\int P(x) S(x+z) d x
$$

It provides some information about $B$, but it is not the same thing. When we substitute Eq. (1) into Eq.( 2):

$$
C(z)=\iint B(u) S(x-u) S(x+z) d u d x
$$

then we see that the integral incorporates any natural broadening present in $S$, magnified by the double integration. So, definitely, $C \neq B$. The equality would hold only if $S$ consisted of $\delta$-functions instead of the real stellar lines. Sometimes one uses a laboratory spectrum for $S$, which has less broadening, although this is still not a bunch of $\delta$ functions. We prefer to work with the observed spectra of standards as this immediately relates the RV zero points of our observations to a standard system and permits inclusion of even the faintest lines in our spectral window.

The Broadening Function approach is to replace Eq. (1) by a set of over-determined, linear, algebraic equations (Ppaper-VII). Thus, the equation takes the shape:

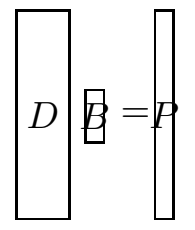

where the rectangular array $D(m, n-m)$ is constructed from appropriately shifted spectrum $S(n)$. In our case, the vectors $P$ and $S$ have dimension of $n=1024$ (the old Thomson chip) or $n=2048$ (the new Marconi chip) while the vector $B$ has a selectable dimension of typically $m=100$ to 200 pixels. The size of $m$ is adjusted to have the broadening function well determined with some baseline around it, e.g. for $m=200$ and the step in velocities of $5.7 \mathrm{~km} / \mathrm{s} / \mathrm{pix}$, the overall span in velocities 
is $\pm 570 \mathrm{~km} / \mathrm{s}$ (see the figures later on). For $n=2048$ and $m=200$, the system of equations have a 10-fold over-determinacy. Selection of $m$ is somewhat critical, as a smaller $m$ leads to better definition of $B$. It is useful to run a $\mathrm{CCF}$ on the $P$ and $S$ spectra first, see where the combined peak is located, select $m$ and then determine $B$.

Any least-squares approach can be used to solve the linear equations thus formed. We utilize the SVD as it has many advantages: (1) Several program spectra can be analyzed for individual $B$ 's using one inverse of the array $D ;(2)$ The role of the featureless continuum leading to linearly dependent equations is automatically diminished in the solution; (3) Existing SVD algorithms are very efficient even for large sets of equations, such as ours. We do not go into the details here and refer the reader to Paper-VII ${ }^{2}$. We did not publish the BF's for individual systems initially and started doing so in Paper-III to illustrate the mounting evidence that many of the program binaries had spectroscopic companions. Thanks to the BF approach we could see third components previously entirely blurred in any CCF attempts.

\section{The main results}

We publish radial velocity data and preliminary orbital solution in bunches of ten orbits per paper, so that we have the ratio: papers/stars $=0.1$. This was a decision made early, in response to the perceived overabundance of single-star studies. This way we can work with individual stars at a reasonable pace producing about 3 papers in about two years. We have been observing our targets at random, as they were visible and once a star is sufficiently covered in the orbital phases, we follow with the BF work and attempted an orbital solution. Frequently surprises happen at that stage: Sometimes the phase coverage is inadequate, sometimes a third spectroscopic component become visible. We delayed analysis of the triples in the first papers, but as their numbers climbed up, we had to deal with them on a routine basis. We also have a few even more difficult close quadruples, to be discussed for the fisrt time in Paper-XI (Pribulla at al., in preparation).

The observing lists have evolved over time. At first, we published systems which Wen Lu liked to observe and analyze... Then, we slowly added more recently discovered, Hipparcos binaries with small photometric amplitudes. Some of these were systems with small orbital inclinations, but most turned out to have companions diluting the binary signal. Frequently, without the BF approach, such binaries would

\footnotetext{
${ }^{2}$ See also: http://www.astro.utoronto.ca/ rucinski/SVDcookbook.html
} 

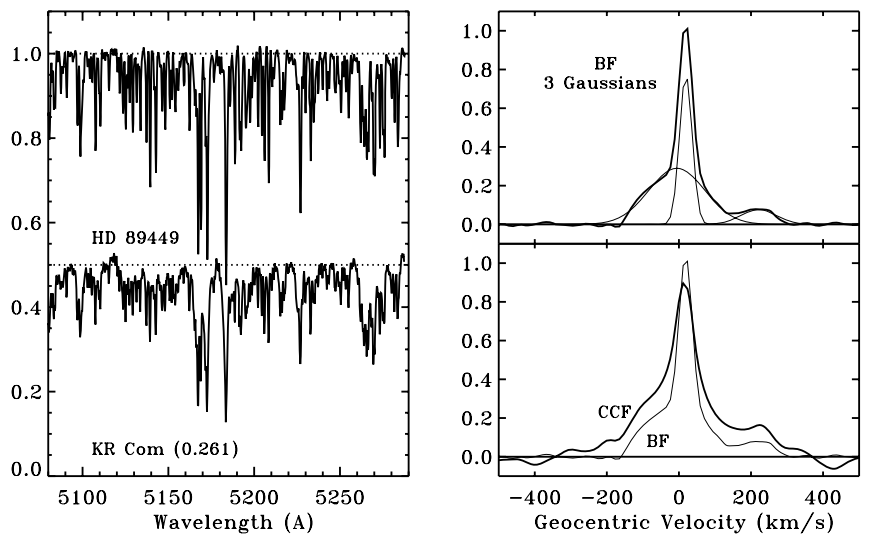

Figure 1. The left panel: The spectrum of a triple system KR Com and of a standard. Although the companion is fainter than the close binary, its sharp line spectrum masks the close binary spectrum which only produces the strange depressed continuum, perhaps interpretable as a spectrum of a super metal-reach star. The right panel: The $\mathrm{BF}$ and its decomposition into the three stars and a comparison of the $\mathrm{BF}$ with the CCF. Because the BF's are linear functions, the light contribution of the 3-rd component can be easily determined by a simple integration, contrary to the CCF requiring calibration of relative intensities.

be called strange and intractable. The example from our Paper VI is shown in Figure 1. Many small amplitude short-period variables discovered by Hipparcos and classified as $\delta$ Sct type turned out to be binaries with $P=2 \times P_{H I P}$. A much smaller number of suspected Hipparcos binaries have been found to be pulsating stars (FH Cam, CU CVn, CC Lyn).

The main result of our survey are - of course - the radial velocity orbits, now available for 100 close binary system; in that more than $3 / 4$ of all are contact binaries. We see many new photometric solutions in the literature driven by the availability of the new spectroscopic mass ratios. As we have stressed many times, in very many cases, $q_{\text {spec }}$ is very far from $q_{p h o t}$, putting in question attempts to find the latter, particularly for partially eclipsing systems.

We hope that one day we will see models of the BF's. Early attempts (Rucinski, 1992; Rucinski and Lu, 1993; Rucinski et al., 1993) should be replaced by a more rigorous approach. A lot of information is there as can be seen for the W-type contact system SW Lac (Paper X) where the increased surface brightness of the secondary is directly visible (Figure 2). We are not satisfied with our preliminary Gaussian fits, but 


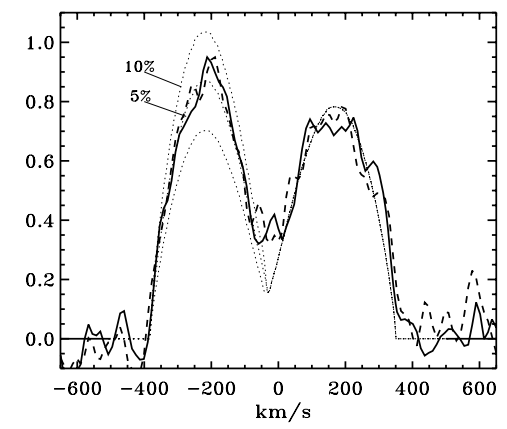

Figure 2. The broadening function at the orbital phase 0.25 for SW Lac. Note that the $\mathrm{BF}$ can be reproduced by a $5 \%$ increase in the secondary effective temperature (dotted lines). A signature of the third component, only very weakly showing up between the two peaks of the contact binary is relatively well defined when all spectra are analyzed together (see the end of this section).

consider them adequate for the first-time estimates of the orbital semiamplitudes $K_{i}$. Theo Pribulla, has recently suggested that rotational profiles give better, more consistent results (notwithstanding the fact that they cannot reproduce asymmetries in the BFs).

We do not see any preference for specific mass ratios in contact binaries; the distribution of $q$ does not seem to peak at any specific value. The smallest $q$ discovered by us for SX Crv is $q=0.066$ (Paper V, see Figure 3) while the largest is $q=0.970$ for V753 Mon (Paper III). The case of SX Crv is of course particularly interesting as a second system (in addition to AW UMa) below the theoretically predicted low limit on the mass ratio (Rasio, 1995). The light curves of SX Crv (an example is shown in Figure 4 (Zola et al., 2004)) do not carry any information on the mass ratio.

As mentioned before, we see many triple systems among our shortperiod binaries. This has prompted two recent investigations, both to be published soon: (1) Theo Pribulla and I have looked at the current numbers of triples among contact binaries, simply as they are, without analysis of biases, selection effects or types of companions which could not be discovered. Depending how we look at the data and what technique (astrometric, spectroscopic, etc.) we use, the lower limit to the frequency of companions comes out at the level $50 \%$ to 65\%. (2) Caroline D'Angelo, Marten van Kerkwijk and I re-analyzed the spectra of the current DDO program by stacking them together, to average out signatures of the rapidly moving close binary and to strengthen the signature of the tertiary; this led to discovery of several faint companions. Both studies are consistent with the possibility that all contact binaries originate in multiple systems. 


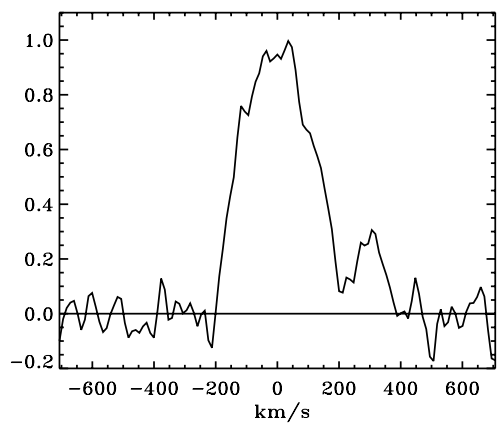

Figure 3. The BF for SX Crv at the orbital phase of 0.25 . Note that the secondary, at $q=0.066$, is easily detectable in spite of its very low luminosity. Paradoxically, for for very small $q$, it is the heavily-broadened primary peak in the BF which must be properly measured for RV shifts as it carries most of information on $q=K_{1} / K_{2}$.

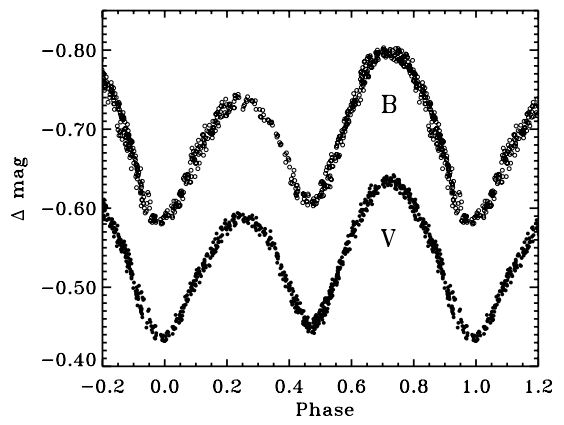

Figure 4. The light curve of SX Crv (from Zola et al.) does not contain any indication that the binary has a very small mass ratio. Instead, a large asymmetry in light maxima is clearly visible. Without spectroscopic information, this system would be basically "unsolvable".

We estimate that we still have some 40 bright $(<10$ mag) binaries with periods shorter than one day at $\delta>-15$ to analyze within our program. Then, we can explore longer periods or observe fainter, very short period $(<0.3$ day) binaries in an attempt to bring our sample closer to a volume limited one.

\section{Acknowledgements}

This paper is written partly to acknowledge contribution of, and express thanks to, many individuals: In addition to the participation of Hilmar Duerbeck and Wen $\mathrm{Lu}$ at the important initial stages, the program enjoyed enthusiastic support of several observers from the Department of Astronomy and Astrophysics of the University of Toronto (Mel Blake, 
Chris Capobianco, George Conidis, Heide DeBond, Stefan Mochnacki, Wojtek Pych, Jim Thomson), from Greece (Kosmas Gazeas), Poland (Piotr Ligeza, Waldek Ogloza, Piotr Rogoziecki, Greg Stachowski) and Slovakia (Theo Pribulla). I would like to thank all of them for their excellent work and contribution to one of the main legacies of the David Dunlap Observatory.

\section{References}

Lu, W. and Rucinski, S. M. Radial Velocity Studies of Close Binary Stars. I. Astron. J., 118, 515-526, 1999.

Lu, W., Rucinski, S. M. and Ogloza, W. Radial Velocity Studies of Close Binary Stars. IV. Astron. J., 122, 402-412, 2001.

Pych, W., Rucinski, S. M., DeBond, H., Thomson, J. R., Capobianco, C. C., Blake, R. M., Ogloza, W., Stachowski, G., Rogoziecki, P., Ligeza, P. and Gazeas, K. Radial Velocity Studies of Close Binary Stars. IX. Astron. J., 127, 1712-1719, 2004.

Rasio, F. A. The Minimum Mass Ratio of W Ursae Majoris Binaries. Astroph. J., 444, L41-L43, 1995.

Rucinski, S. M. Spectral-Line Broadening Functions of W UMa-type Binaries. I. AW UMa. Astron. J., 104, 1968-1981, 1992.

Rucinski, S. M. Radial Velocity Studies of Close Binary Stars. VII. Astron. J., 124, 1746-1756, 2002.

Rucinski, S. M. and Lu, W. Spectral-Line Broadening Functions of W UMa-type Binaries. II. AH Vir Astron. J., 106, 361-371, 1993.

Rucinski, S. M. and Lu, W. Radial Velocity Studies of Close Binary Stars. II. Astron. J., 118, 2451-2459, 1999.

Rucinski, S. M. and Lu, W. W Crv: The shortest-period Algol with non-degenerate components? Mon. Not. Roy. Astr. Soc., 315, 587-594, 2000.

Rucinski, S. M., Lu, W. and Shi, J. Spectral-Line Broadening Functions of W UMatype Binaries. III. W UMa. Astron. J., 106, 1174-1180, 1993.

Rucinski, S. M., Lu, W. and Mochnacki, S. W. Radial Velocity Studies of Close Binary Stars. III. Astron. J., 120, 1133-1139, 2000.

Rucinski, S. M., Lu, W., Mochnacki, S. W., Ogloza, W. and Stachowski, G. Radial Velocity Studies of Close Binary Stars. V. Astron. J., 122, 1974-1980, 2001.

Rucinski, S. M., Lu, W., Capobianco, C. C., Mochnacki, S. W., Blake, R. M., Thomson, J. R., Ogloza, W., and Stachowski, G. Radial Velocity Studies of Close Binary Stars. VI. Astron. J., 124, 1738-1745, 2002.

Rucinski, S. M., Capobianco, C. C., Lu, W., DeBond, H., Thomson, J. R., Mochnacki, S. W., Blake, R. M., Ogloza, W., Stachowski, G. and Rogoziecki, P. Radial Velocity Studies of Close Binary Stars. VIII. Astron. J., 125, 3258-3264, 2003.

Rucinski, S. M., Pych, W., Ogloza, W., DeBond, H., Thomson, J. R., Mochnacki, S. W., Capobianco, C. C., Conidis, G. and Rogoziecki, P. Radial Velocity Studies of Close Binary Stars. X. Astron. J., 130, 767-775, 2005.

Zola, S., Rucinski, S. M., Baran, A., Ogloza, W., Pych, W., Kreiner, J. M., Stachowski, G., Gazeas, K., Niarchos, P., Siwak, M. Physical Parameters of Components in Close Binary Systems: III. Acta Astr., 54, 299-312, 2004. 